\title{
Tongue coating microbiome regulates the changes in tongue texture and coating in patients with post-menopausal osteoporosis of Gan-shen deficiency syndrome type
}

\author{
WENNA LIANG ${ }^{1}$, XIHAI LI ${ }^{2}$, YACHAN LI ${ }^{1}$, CANDONG LI $^{1}$, BIZHENG GAO ${ }^{1}$, HUIJUAN GAN $^{1}$, SUMIN LI $^{3}$, \\ JIANYING SHEN $^{1}$, JIE KANG ${ }^{1}$, SHANSHAN DING ${ }^{1}$, XUEJUAN LIN ${ }^{1}$ and LINGHONG LIAO ${ }^{1}$ \\ ${ }^{1}$ Research Base of Traditional Chinese Medicine Syndrome; ${ }^{2}$ Academy of Integrative Medicine, \\ Fujian University of Traditional Chinese Medicine, Fuzhou 350122; ${ }^{3}$ The Affiliated People's Hospital, \\ Fujian University of Traditional Chinese Medicine, Fuzhou 350004, P.R. China
}

Received June 2, 2013; Accepted September 2, 2013

DOI: $10.3892 /$ ijmm.2013.1485

\begin{abstract}
Tongue inspection is a unique and important method of diagnosis in traditional Chinese medicine (TCM). It is a daignostic approach which involves observing the changes in the tongue proper and tongue coating in order to understand the physiological functions and pathological changes of the body. However, the biological basis of TCM tongue diagnosis remains to be poorly understood and lacks systematic investigation at the molecular level. In this study, we evaluated the effects of tongue coating microbiome on changes in the tongue texture and coating in patients with post-menopausal osteoporosis (PMO) of Gan-shen deficiency syndrome type. Our aim was to delineate the mechanisms of tongue coating microbiome-induced changes in the tongue texture and coating by investigating the histomorphological changes and performing a bacterial analysis of the tongue coating. We found that the number of intermediate cells in the red tongue with a thin coating was higher, while the number of superficial cells in the red tongue with a thin coating was lower. The maturation value (MV) of tongue exfoliated cells in the red tongue with a thin coating decreased, compared with that in the pale red tongue with a thin white coating. Furthermore, the total bacterial count, oral streptococcus, Gram-positive $\left(\mathrm{G}^{+}\right)$and Gram-negative $\left(\mathrm{G}^{-}\right)$ anaerobic bacteria in the red tongue with a thin coating was significantly decreased compared with the pale red tongue with a thin white coating. The results of ultrastructural examination demonstrated that the number of epithelial cells and bacteria in the red tongue with a thin coating decreased compared with that in the pale red tongue with a thin white coating. These
\end{abstract}

Correspondence to: Dr CanDong Li, Research Base of Traditional Chinese Medicine Syndrome, Fujian University of Traditional Chinese Medicine, 1 Huatuo Road, University Town, Minhou Shangjie, Fuzhou 350122, P.R. China

E-mail: fjzylcd@126.com

Key words: microbiome, bacterium, tongue coating, post-menopausal osteoporosis, traditional Chinese medicine observations indicate that the tongue coating microbiome may be an important factor contributing to changes in the tongue in patients with PMO of Gan-shen deficiency syndrome type.

\section{Introduction}

Traditional Chinese medicine (TCM) has been widely used for the treatment of various diseases in East Asian countries for at least 3,000 years, and it is also an alternative and complementary medical system in Western countries. TCM takes a more holistic approach to medicine with emphasis on syndrome differentiation and treatment and the integrity of the human body, including the close association between humans and their natural and social environment $(1,2)$. TCM diagnosis is based on the information collected from four diagnostic processes, namely, looking, smelling and listening, asking and touching. The most common tasks are to inspect the tongue and take the pulse during the process of clinical diagnosis and treatment. Determining the health status of the internal organs of patients by inspecting the tongue has been a common method of TCM practitioners for thousands of years. Tongue coating, one of the direct objective foundations for TCM clinical diagnosis and treatment, plays a crucial role in reflecting the occurrence, development and prognosis of disease; thus, it is of great importance for syndrome differentiation, predicting prognosis, determining the type of treatment and prescribing a formula $(3,4)$.

TCM applies various therapeutic methods to promote the resistance of the body to diseases, according to Zheng (also known as TCM syndrome) in TCM. Zheng, an essential and integral part of TCM theory, is a characteristic profile of all clinical manifestations which can be judged by TCM practitioners. In TCM, all diagnostic and therapeutic methods are based on the differentiation of Zheng, which is an outcome after analyzing all symptoms and signs (including inspecting the tongue and taking the pulse) (5). TCM practitioners differentiate the Zheng of individual patients to be used as a holistic summary of the health status of the patient and is determined mainly by the color and texture of the tongue coating (6). The tongue coating is connected with many parts of the 
human body, such as the internal organs and the meridians. Therefore, it is very important and useful during examination for confirming TCM diagnosis as it can present strong visual indicators of the overall mental and physical disharmony or harmony of the individual (7). The tongue is divided into the tongue root, tongue center, tongue margins and tongue tip. Fig. 1A illustrates each section of the tongue and its correspondence to different internal organs according to TCM. The bilateral sides of the tongue reflect the pathological changes in the liver and gallbladders, while the kidneys are mirrored by the tongue root. The pathological changes in the heart and lungs are reflected by the tongue tip, while changes in the spleen and stomach correspond to the center of tongue.

Post-menopausal osteoporosis (PMO), defined as a generalized skeletal disorder due to deteriorating bone quality or decreased bone density, is the most common cause of age-related bone loss $(8,9)$. Estrogen deficiency in post-menopausal women may lead to an increase in bone breakdown related to a decrease in bone mass and the degeneration of the bone microstructure. The majority of women $>50$ years suffer from osteoporotic fractures during their lifetime (10). PMO is an important contributor to the global burden of non-communicable disease and a major public health issue, which belongs to the category of Gu Wei in TCM theory. The majority of PMO cases belong to the Gan-shen deficiency syndrome type, a typical clinical characteristic of which is a red tongue with a thin coating.

In modern Western medicine, the tongue may be used to provide crucial clues as to the current condition of the human body and global information as to the health status of an individual and has increasingly been viewed as an extension of the upper gastrointestinal tract. Tongue diagnosis is more convenient for patients, as well as doctors compared with other diagnostic regimens, such as tissue biopsies or blood tests (11). Therefore, studying the molecular bases of tongue diagnosis is important for understanding this long-standing medical practice and may be a promising contribution to personalized medicine. In the present study, we hypothesized that tongue coating microbiome affects the changes in the tongue in patients with PMO of Gan-shen deficiency syndrome type. We carried out a histomorphological examination and bacterial analysis of the tongue coating and investigated the possible mechanisms mediating the changes in the tongue. We found that the amount of epithelial cells and bacteria in the red tongue with a thin coating was lower than that in the pale red tongue with a thin white coating, which was accompanied by histomorphological changes in tongue exfoliated cells, demonstrating that the tongue coating microbiome may be a factor contributing to changes in the tongue.

\section{Materials and methods}

General materials. Between January 2012 to March 2013, a total of 90 patients with PMO and 30 healthy women from the Second People's Hospital of Fujian Province were enrolled in this study. Patient materials were recorded in a uniform data collection table. There was no significant difference in age between the patients with PMO $(60.89 \pm 4.57)$ and the healthy women (57.56 \pm 3.84$)(\mathrm{P}>0.05)$, hence comparability.

Inclusion criteria. Women who were post-menopausal for at least one year and had osteopenia or osteoporosis as evidenced by a bone mineral density of $<2.5 \mathrm{SD}$ or fragility fractures according to previously described criteria (12), were included in this study.

The Gan-shen deficiency syndrome type was differentiated in reference to the standard for syndromes and the Guiding Principles of Clinical Research on New Drugs of Traditional Chinese Medicine, namely, soreness of the waist and knees; back pain; dysphoria with a feverish sensation in the chest, palms and soles of the feet; light-headedness; insomnia and dreaminess; a dry throat and tongue; a red tongue with a thin coating (Fig. 1C) and pulse count. This study was approved by the Medical Ethics Committee of the Second People's Hospital of Fujian Province and all patients and healthy women signed informed consent forms.

Exclusion criteria. The exclusion criteria included patients with deranged renal function, abnormal thyroid function, significant liver disease, a history of cancer, patients who had received regular therapy with a phosphate-binding antacid, estrogen replacement therapy within the previous 9-12 months, therapy with any other drug that affects the skeleton, such as steroids, anti-convulsants and anticoagulants; patients who had been treated with any other method which can influence the observation of indexes in this research; and patients who gave up the observation halfway and refused to objectively fill in the indexes in this study.

Collection of exfoliated cells. The tongues of patients and healthy women were judged by two TCM doctors from between 8-9 a.m. All healthy women exhibited a normal tongue (pale red with a thin white coating, Fig. 1B). All subjects were asked to rinse their mouths gently with saline solution. Tongue exfoliated cells were scraped from the tongue fur and immediately smeared on pre-cleaned microscopic slides or collected in a $1.5 \mathrm{ml}$ EP tube.

Histomorphological examination of tongue coating. The smears were separately fixed with $4 \%$ paraformaldehyde solution (pH 7.3) and 95\% alcohol for $15 \mathrm{~min}$ and stained with hematoxylin and eosin (H\&E) and Papanicolaou (Pap) to assess the general morphology. All the slides were observed under a light microscope using low magnification. The maturation index (MI) and the proportions of parabasal to intermediate to superficial cells were recorded for each specimen as previousy described (13) using the following formula: maturation value $(\mathrm{MV})=$ parabasal cells $\mathrm{x} 0+$ intermediate cells $\mathrm{x} 0.5+$ superficial cells x 1.0.

Analysis of tongue coating bacteria. Tongue coating $(0.1 \mathrm{~g})$ was scraped from the middle of the tongue using a sterile curette, shocked and diluted 10 times from $10^{-1}-10^{-6}$. Ten $\mu 110^{-4}-10^{-6}$ diluted solution was evenly smeared onto two dishes and incubated for $48 \mathrm{~h}$ in an anaerobic box. Anaerobic bacteria isolation used the triple scale identification of clinical anaerobic bacteria. The numbers of bacteria were detected by $\log$ (colony-forming units, $\mathrm{CFU} / \mathrm{g}$ ).

Holistic morphology of tongue coating. The smears of the tongue coating were fixed with $2 \%$ glutaraldehyde solution, washed with $0.1 \mathrm{M}$ sodium cacodylate buffer and post-fixed 

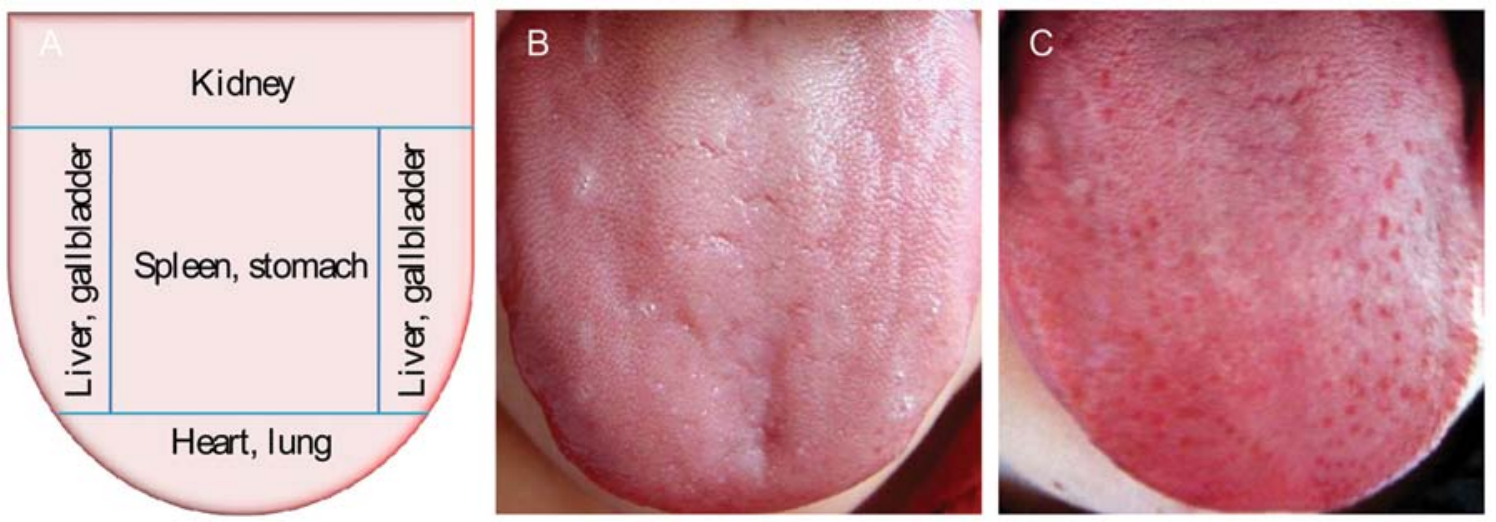

Figure 1. Traditional Chinese medicine (TCM) tongue diagnosis and tongue coating appearance classification. (A) Sections of the tongue and their correspondence to internal organs in TCM; (B) a pale red tongue with a thin white coating represents a healthy individual; (C) a red tongue with a thin coating represents a patient with Gan-shen deficiency syndrome type.
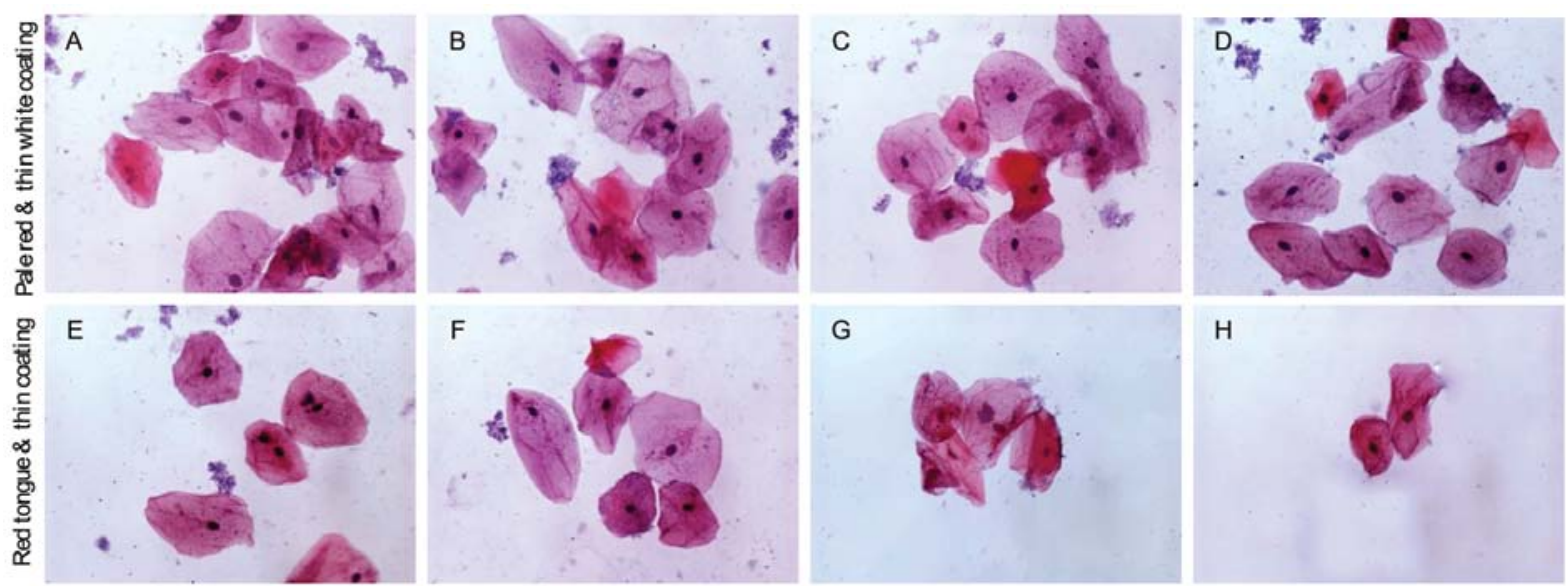

Figure 2. Histological analysis of tongue exfoliated cells. Smears of the tongue coating stained with H\&E, illustrating the histological changes. The epithelial cells from the pale red tongue with a thin white coating revealed that the largest cells are flattened and oval in shape with a low nucleus to cytoplasm (N/C) ratio and small and oval to pyknotic nuclei (A-D, x200). The smears from the red tongue with a thin coating showed a few epithelial cells with a clear background (E-H, x200).

with $1 \%$ osmium tetroxide. After dehydrating with an alcohol gradient series and dehydrating with isoamyl acetate again, the smears were dried using a critical point dryer (HCP-2; Hitachi, Tokyo, Japan). After being coated with a layer of gold, all smears were observed under a scanning electron microscope (SEM, JSM-6380LV; Jeol, Tokyo, Japan).

Ultrastructural examination of tongue coating. The precipitation of tongue coating was fixed in $3 \%$ glutaraldheyde and $1.5 \%$ paraformaldehyde solution $(\mathrm{pH} 7.3)$ at $4^{\circ} \mathrm{C}$ for $24 \mathrm{~h}$, post-fixed with $1 \%$ osmic acid and $1.5 \%$ potassium hexacyanoferrate (II) solution $\left(\mathrm{pH} \mathrm{7.3)}\right.$ at $4^{\circ} \mathrm{C}$ for $2 \mathrm{~h}$. The samples were then washed, dehydrated with graded alcohol and embedded in Epon-Araldite resin. Ultra-thin sections were cut on a Leica ultramicrotome and stained $2 \%$ aqueous uranyl acetate, counterstained with $0.3 \%$ lead citrate and examined under a transmission electron microscope (TEM, H7650; Jeol).

Safety estimation. The symptoms and physical signs of adverse reactions that occurred were recorded and routine tests on blood, urine and stool, liver and renal functions were performed, as well as an electrocardiogram (ECG).
Statistical analysis. All data are represented as the means \pm standard deviation (SD) and analyzed using the SPSS package for Windows (version 13.0). Statistical analysis of the data was performed using a paired samples t-test. A value of $\mathrm{P}<0.05$ was considered to indicate a statistically significant difference.

\section{Results}

Histomorphology of tongue exfoliated cells. The epithelial cell types were readily identified and their proportions in the tongue exfoliated cell smears were applied in the characterization of changes in the tongue coating. Superficial cells, the largest cells, were flattened and oval in shape with a low nucleus to cytoplasm (N/C) ratio and small and oval to pyknotic nuclei. As shown in Fig. 2, the morphology of intermediate cells varied in size from small and medium to large, but was no larger than the superficial cells. Intermediate cells wer round to oval in shape with round and slightly flattened vesicular nuclei and smooth cytoplasmic and nuclear membranes. The parabasal epithelial cells, characterized by a high N/C ratio, were the thickest and smallest cells with 

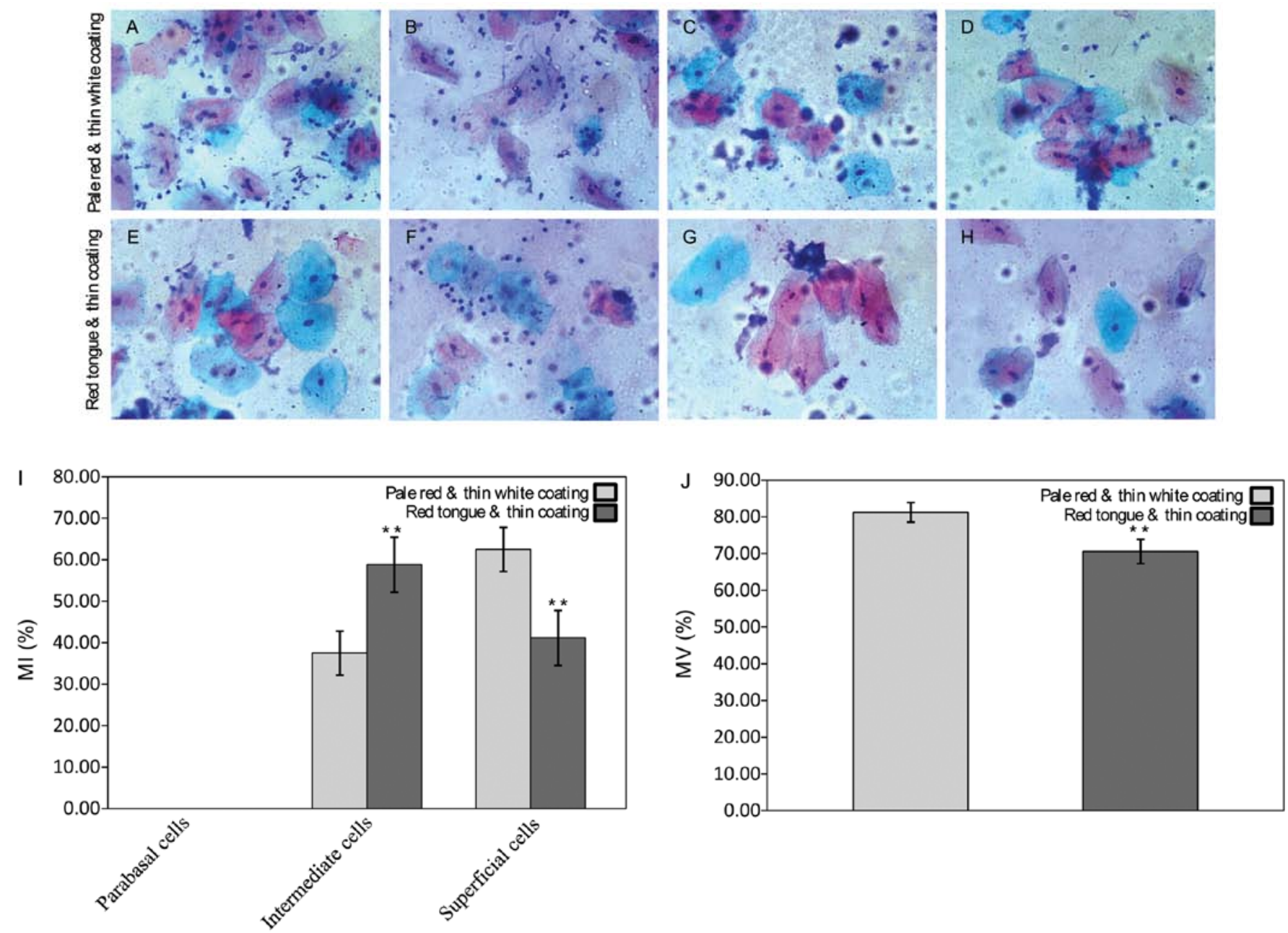

Figure 3. Changes in keratinocyte of tongue exfoliated cells. Tongue exfoliated cells with Pap staining are mainly classified into parabasal, intermediate and superficial cells. The parabasal cells are mainly round in shape with apparently spherical nuclei and the cytoplasm is dyed bright blue. The intermediate cells are varied shape (round, oval and boat) and the cytoplasm is dyed blue. The superficial cells (the largest cells) are flattened and oval in shape with small and oval to pyknotic nuclei and the cytoplasm is dyed red. The pale red tongue with a thin white coating was composed of keratinocytes and a few intermediate cells (A-D, x200). The red tongue with a thin coating consisted mature keratinocytes and many intermediate cells (E-H, x200). (I and J) The maturation index (MI) and maturation value (MV) of the tongue exfoliated cells were calculated to identify the proportions of parabasal to intermediate to superficial cells. Data are $\pm \mathrm{SD}$ (error bars), ${ }^{* *} \mathrm{P}<0.01$ vs. pale red tongue with thin white coating.

apparently spherical nuclei and smooth, rounded cytoplasmic and nuclear membranes.

changes in MI and MV in the tongue exfoliated cells in patients with PMO of Gan-shen deficiency syndrome type. Pap staining is mainly used for the analysis of changes in keratinocyte in the tongue coating. Keratinocytes according to the degree of differentiation are classified into precornified cells, immature keratinocytes and mature keratinocytes. The pale red tongue with a thin white coating was mostly composed of keratinocytes, including some immature keratinocytes and mature keratinocytes and a few intermediate cells (Fig. 3A-D). The smears from the red tongue with a thin coating had a clearer background than those from the pale red tongue with a thin white coating. The number of epithelial cells in the red tongue with a thin coating decreased and mainly consisted of mature keratinocytes and many intermediate cells (Fig. 3E-H).

MI (the percentage of parabasal to intermediate to superficial cells) and MV are indicators in the pathophysiology of tongue exfoliated cells and indicate the maturity of epithelial cells. The number of intermediate cells in the red tongue with a thin coating $(58.83 \pm 6.62 \%)$ was higher than that in the pale red tongue with a thin white coating $(37.50 \pm 5.32 \%, \mathrm{P}<0.01)$. The number of superficial cells in the red tongue with a thin coating $41.17 \pm 6.62 \%$ was lower than that in the pale red tongue with a thin white coating $(62.50 \pm 5.32 \%, \mathrm{P}<0.01)$ (Fig. 3I). The $\mathrm{MV}$ of the tongue exfoliated cells $(70.58 \pm 3.31 \%)$ from the red tongue with a thin coating was significantly decreased compared with that from the pale red tongue with a thin white coating $(81.25 \pm 2.66 \%, \mathrm{P}<0.01)$ (Fig. $3 \mathrm{~J})$, suggesting that the red tongue with a thin coating mainly consisted of intermediate cells.

Tongue coating microbiome-induced changes in patients with PMO of Gan-shen deficiency syndrome type. Bacteria from the pale red tongue with a thin white coating and from the red tongue with a thin coating consisted of oral streptococci, Veillonella, Gram-positive $\left(\mathrm{G}^{+}\right)$anaerobic bacteria, Gram-negative $\left(\mathrm{G}^{-}\right)$anaerobic bacteria and aerobic bacteria, 


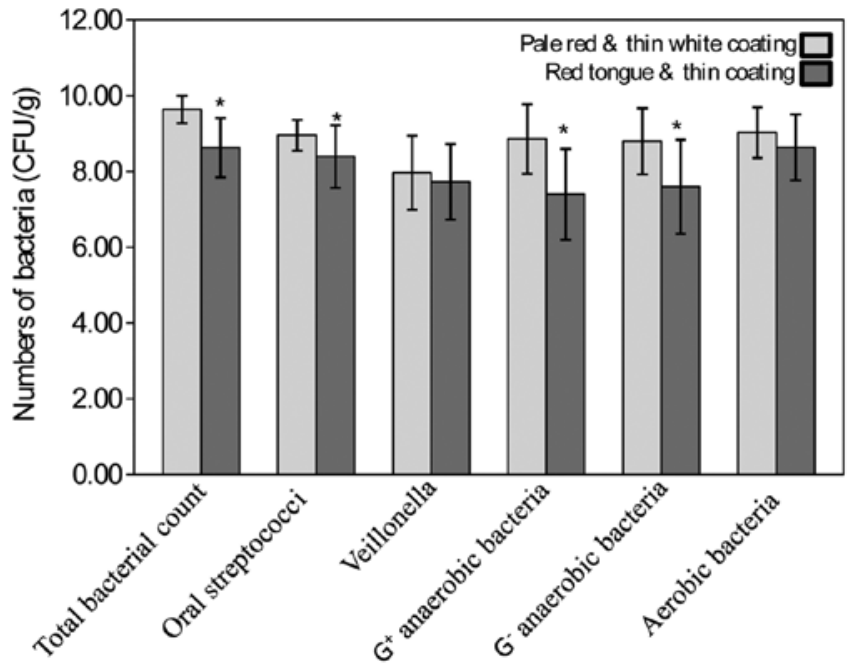

Figure 4. Changes in bacteria in tongue coating. Changes in the amount of bacteria between the pale red tongue with a thin white coating and the red tongue with a thin coating. Shown is the total bacterial count, oral streptococci, $\mathrm{G}^{+}$anaerobic bacteria and $\mathrm{G}^{-}$anaerobic bacteria. Data are presented as the means $\pm \mathrm{SD}$ (error bars), ${ }^{*} \mathrm{P}<0.05$ vs. pale red and thin white coating.

as the main parts of the tongue microbiome. As illustrated in Fig. 4, the total bacterial count in the red tongue with a thin coating $(8.63 \pm 0.78 \mathrm{CFU} / \mathrm{g})$ was significantly decreased compared with that in the pale red tongue with a thin white coating $(9.64 \pm 0.36 \mathrm{CFU} / \mathrm{g}, \mathrm{P}<0.05)$. The amount of oral predominant bacteria (oral streptococci and $\mathrm{G}^{+}$anaerobic bacteria, $8.40 \pm 0.83$ and $7.40 \pm 1.20 \mathrm{CFU} / \mathrm{g}$ ) in the red tongue with a thin coating was lower than that in the pale red tongue with a thin white coating $(8.96 \pm 0.41$ and $8.86 \pm 0.92 \mathrm{CFU} / \mathrm{g}$,
$\mathrm{P}<0.05)$. The amount of $\mathrm{G}^{-}$anaerobic bacteria in the red tongue with a thin coating $7.60 \pm 1.24 \mathrm{CFU} / \mathrm{g}$ was lower than that in the pale red tongue with a thin white coating $(8.80 \pm 0.87 \mathrm{CFU} / \mathrm{g}$, $\mathrm{P}<0.05)$. Thus, the changes in the bacterial count may be an important factor contributing to tongue coating microbiomeinduced changes in the red tongue with a thin coating.

Ultrastructural changes in the tongue coating in patients with PMO of Gan-shen deficiency syndrome type. The tongue coating is mainly composed of exfoliated cells, bacteria, food residuals and mucus. Individual epithelial cells and/or scattered squamous epithelial cells together were visible in the tongue exfoliated cells (Figs. 5 and 6). Coccus and bacillus were the most abundant bacteria in the tongue dorsum microbiome. Tongue exfoliated cells were generally irregular and characterized by many microvilli-like structures and corrugations at the cellular surface (Figs. 7 and 8). The cytoplasm contained few organelles and presented clearly visible tonofilaments. The nuclei of the tongue exfoliated cells were oval in shape with indentations and heterochromatin was observed preferentially at the periphery of the nucleus. Desmosomes providing intercellular adhesive strength required for the integrity of epithelial cells were present in a few tongue exfoliated cells (Fig. 7I-L). Tongue exfoliated cells were surrounded by a large number of cocci and bacilli. The amount of epithelial cells and bacteria in the red tongue with a thin coating was lower than that in the pale red tongue with a thin white coating (Figs. 5-8). Our results revealed that the number of epithelial cells and bacteria in the red tongue with a thin coating significantly decreased, indicating that the tongue coating microbiome plays an important role in changes in the tongues of patients with PMO of Gan-shen deficiency syndrome type.
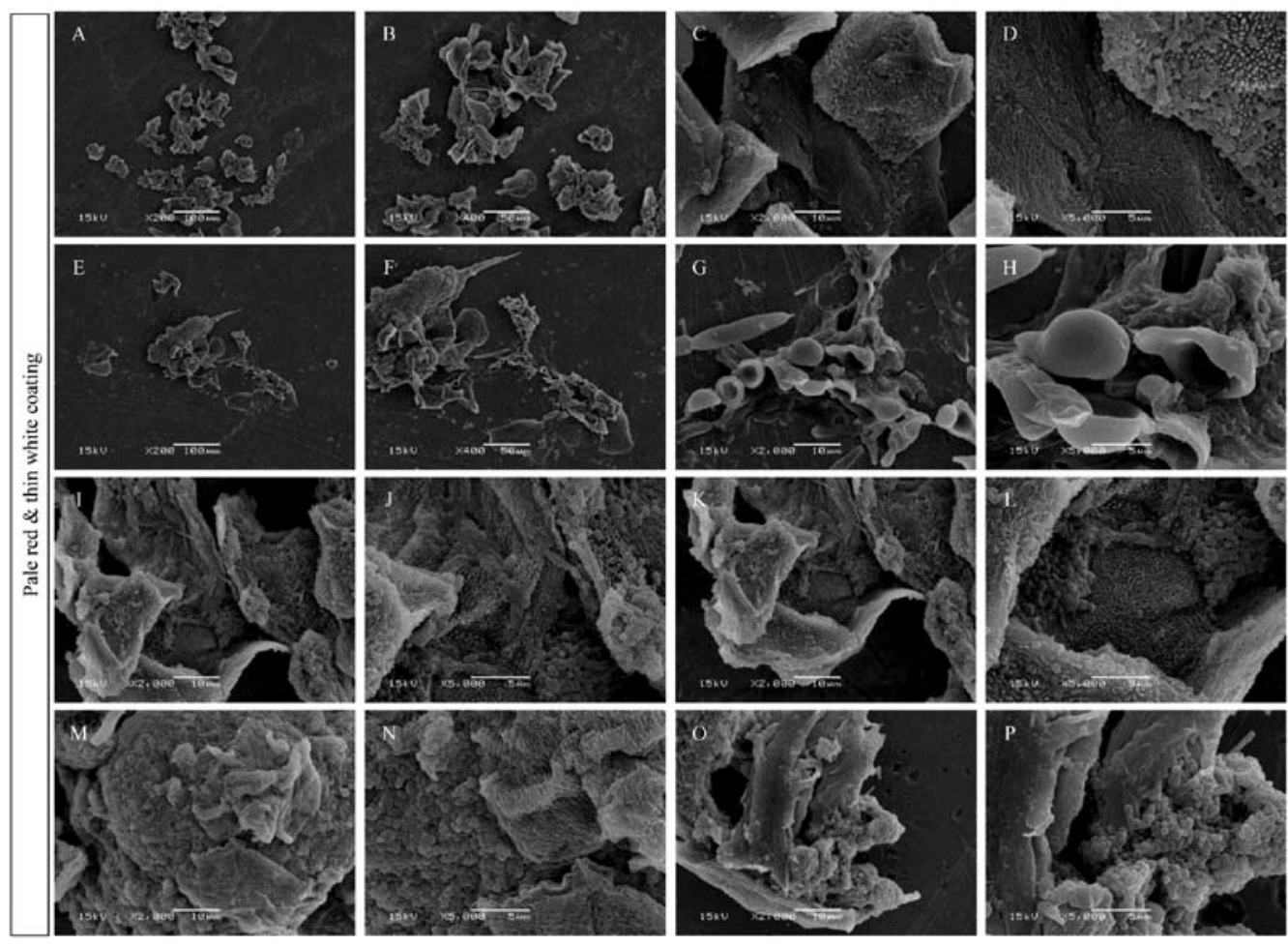

Figure 5. Holistic morphology of pale red tongue with thin white coating. Holistic morphology of tongue coating obtained by a scanning electron microscope (SEM). (A-P) Images of healthy women. Pale red tongue with a thin white coating contained many epithelial cells and bacteria. 

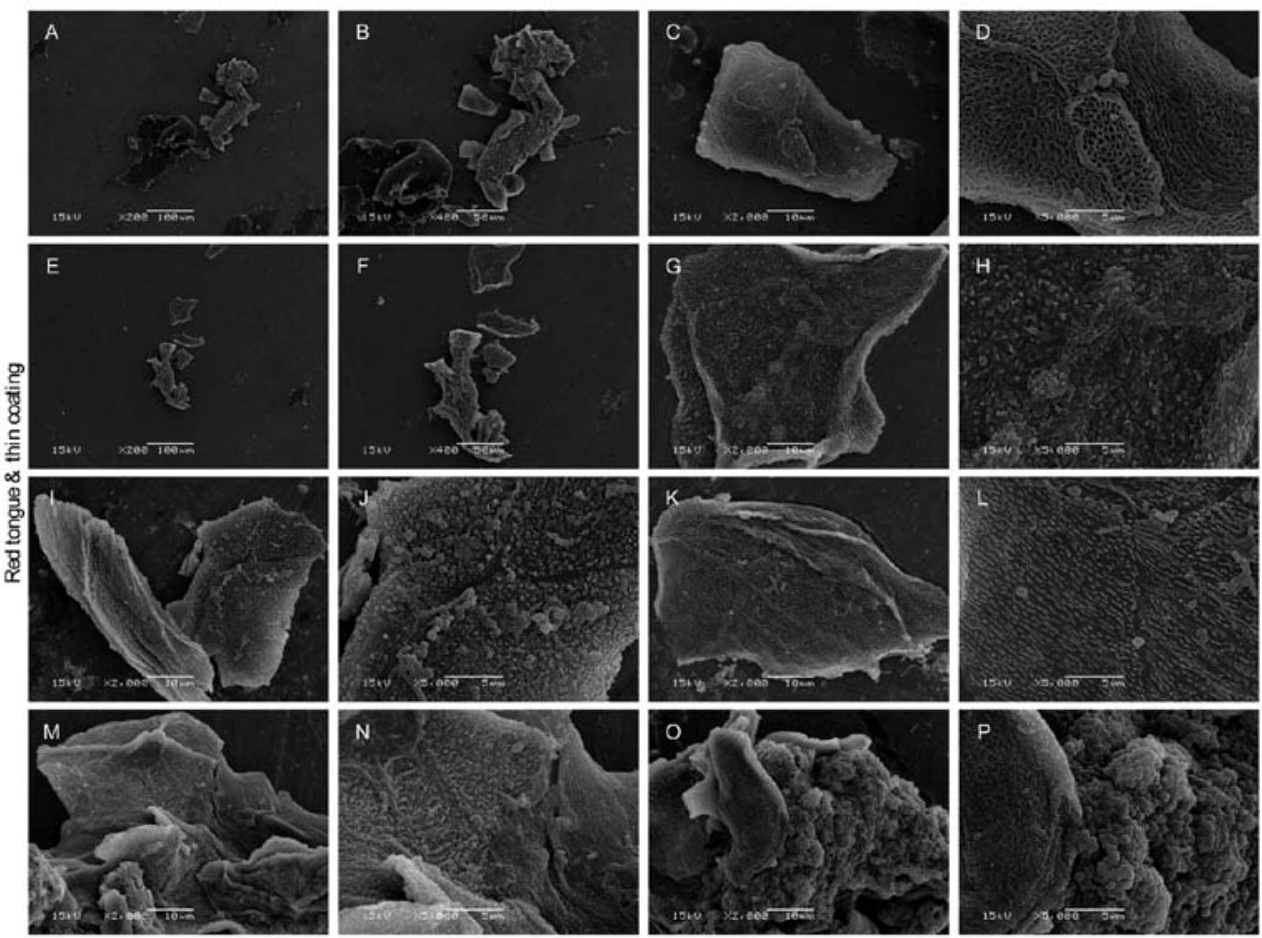

Figure 6. Holistic morphology of red tongue with thin coating. Holistic morphology of tongue coating obtained by a scanning electron microscope (SEM). (A-P) Images of patients with post-menopausal osteoporosis. Red tongue with a thin coating contained a few epithelial cells and bacteria.
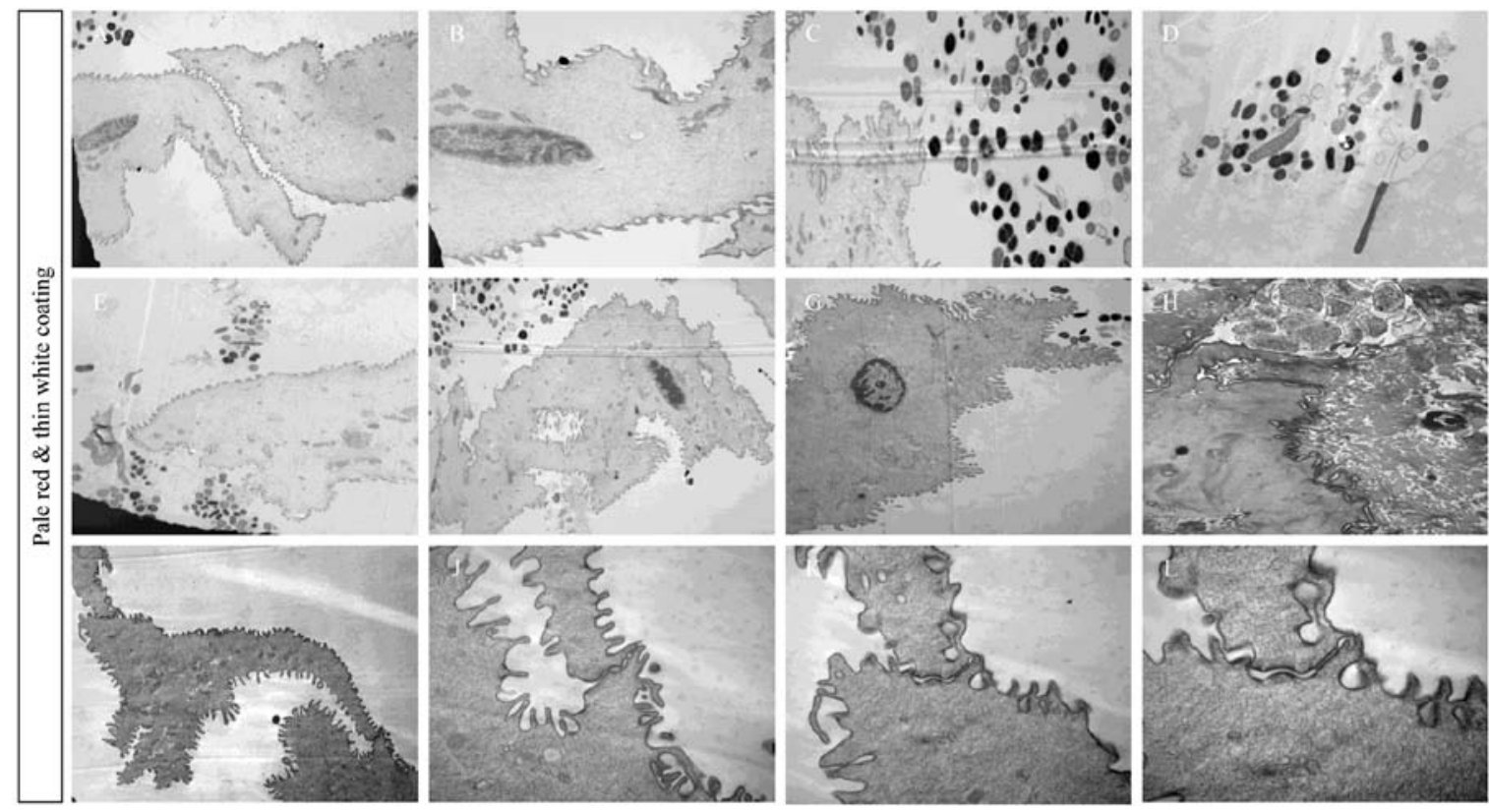

Figure 7. Ultrastructural characteristics of pale red tongue with thin white coating. Ultrastructural characteristics of tongue coating obtained using a transmission electron microscope (TEM). (A-L) Images of healthy women. Pale red tongue with thin white coating contained many tongue exfoliated cells with many microvilli-like structures and corrugations at the cellular surface, as well as abundant bacteria. The nuclei of tongue exfoliated cells had a small oval-like shape with indentations. (I-L) Desmosomes are present between the tongue exfoliated cells. Magnification: (E) x3,000; (A, D and F) x5,000; (C) x7,000; (G and I) x8,000; (B) x15,000; (H, J and K) x30,000; and (L) x50,000.

\section{Discussion}

In the long history of traditional clinical practice in China, TCM practitioners have typically classified patients of the same disease into subtypes as different Zheng from a holistic perspective on the patient overall status. Many factors are used in the discrimination of Zheng; among these the tongue is one of the major factors. It is understood that the human microbiome is associated with a number of diseases and the human health status $(14,15)$. Thus, we hypothesized that changes in the texture and coating of the tongue may mirror features of the tongue microbiome, which is associated with 

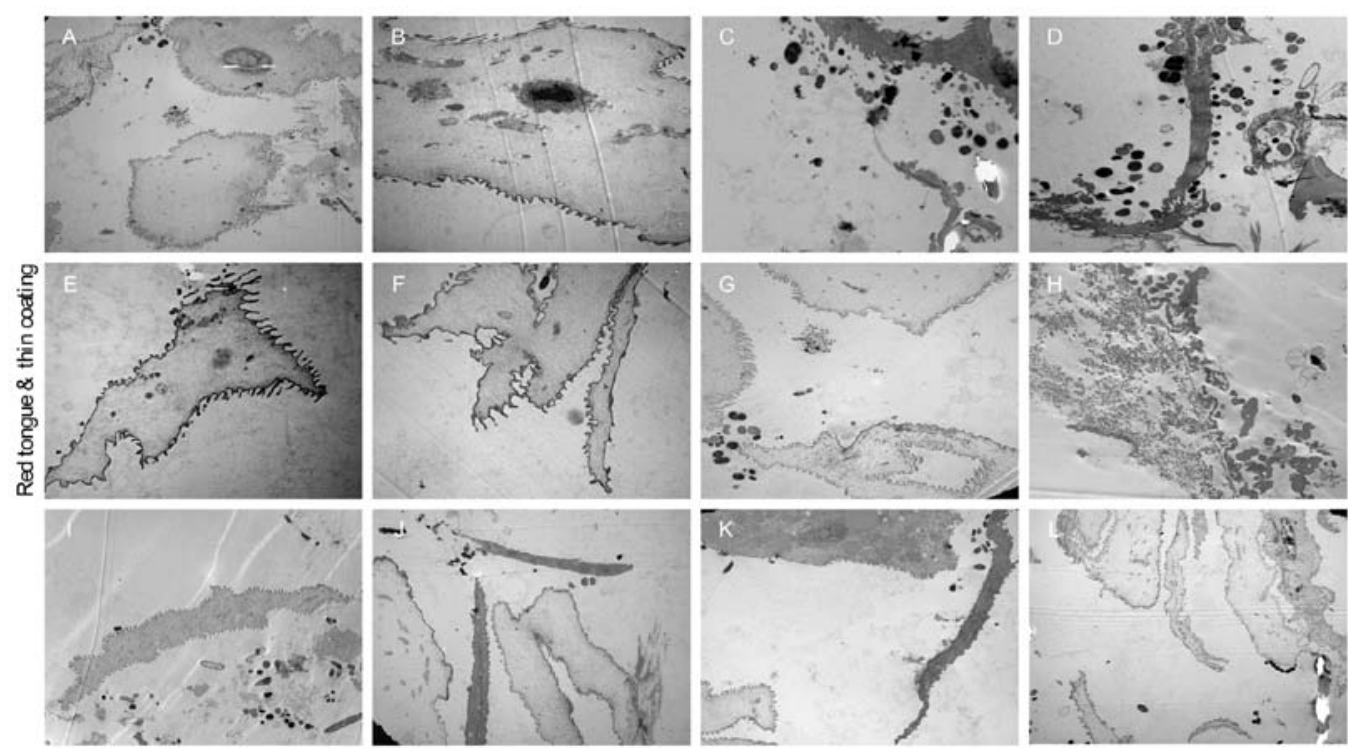

Figure 8. Ultrastructural characteristics of red tongue with thin coating. Ultrastructural characteristics of tongue coating obtained using a transmission electron microscope (TEM). (A-L) Images of patients with post-menopausal osteoporosis. Red tongue with a thin coating contained a few tongue exfoliated cells and bacteria. Magnification: (A and L) x4,000; (C, D, I, J and K) x5,000; (G) x6,000; (B, E and F) x10,000; and (H) x12,000.

the health status of the human body in patients with PMO of Gan-shen deficiency syndrome type. To examine this hypothesis, we performed a bacterial analysis of tongue coating samples from 90 patients with PMO (of Gan-shen deficiency syndrome type) with a red tongue with a thin coating, as well as 30 healthy women with a pale red tongue with a thin white coating. We found that the amount of epithelial cells and bacteria in the red tongue with a thin coating was decreased and this was accompanied by histomorphological changes in tongue exfoliated cells. In addition, the tongue exfoliated cells mainly consisted of intermediate cells in the red tongue with a thin coating. Taken together, our results demonstrate that the tongue microbiome may be a key factor contributing to changes in tongue texture and coating.

Tongue diagnosis, a simple, valuable and non-invasive diagnostic method, has been repeatedly verified by clinical practitioners of TCM. TCM treats tongue appearance as an outer manifestation of the status of the human body by examining the local and general shape, as well as the color of the coating and tongue (16). A pale red tongue with a thin white coating is considered normal tongue according to TCM. Some characteristic changes occur in the tongue during the process of disease diagnosis. Most tongue attributes are on the tongue surface, including color, coating, moisture, size and shape. These signs not only reveal the overall status of the human body, but also correlate with specific disharmonies and organ functions (17). The color and the coating are the two main features of the tongue in Zheng diagnosis. The color of the tongue provides information as to the health status of an individual. For example, a a white tongue suggests the existence of mucus deposits, cold attack or a weakness in the blood and a dark red color indicates ulcerations or inflammation (18). Moreover, blue or purple points out stagnation of the blood circulation and a yellow tongue indicates a disorder of the liver and gallbladder that corresponds to the area of the tongue where the color appears.
The tongue surface is covered with a layer of semitransparent mucosa and the tiny projections on the mucosal fold are termed tongue papillae, which are classified into four types, including the foliate papillae, circumvallate papillae, fungiform papillae and filiform papillae $(19,20)$. The fungiform papillae and filiform papillae are closely associated with the condition of the tongue. The cone-shaped filiform papillae are the most abundant and are distributed over the tongue tip, tongue body and tongue edges. The exfoliated cells, food residuals, bacteria and mucus may fill up in the interspaces of filiform papillae and form the white fur-like substance, termed the tongue coating. The results from the present study demonstrated that the pale red tongue with a thin white coating was mostly composed of keratinocytes and a few intermediate cells; however, the red tongue with a thin coating consisted of mature keratinocytes and many intermediate cells. The MV of tongue exfoliated cells in the red tongue with a thin coating decreased compared with that in the pale red tongue with a thin white coating.

Previous studies have reported that TCM tongue diagnosis is a helpful approach for making a clinical decision $(21,22)$. The term 'geographic tongue' has been used to refer to tongues with discoloured cracks or regions that accompany environmental sensitivity or illness $(23,24)$. In edentate patients, the tongue coating may be an indicator of the risk of aspiration pneumonia, as it is also associated with a number of viable salivary bacteria (25). It has been reported that the microfloral characterization of the tongue coating may be associated with the risk of pneumonia-related health issues in institutionalized older adults (26). Hence, the tongue may have the potential for inferring systemic disorders in clinical diagnoses. However, the biological bases of different tongue coating appearances remains poorly understood and lacks systematic investigation at the molecular level. Therefore, in the present study, we examined whether the tongue coating microbiome affects changes in the tongue. 
In modern Western medicine, the tongue microbiome affecting changes in the tongue is identified as a key factor $(27,28)$. Statistical analyses of the tongue dorsa bacteria have demonstrated the potential connection between the traditional tongue diagnosis and the tongue microbiome $(29,30)$. Compared with the normal tongue dorsa bacteria, we found that the total bacterial count, oral streptococci and $\mathrm{G}^{+}$anaerobic bacteria in the red tongue with a thin coating was significantly decreased. The comparison results were demonstrated by the ultrastructural changes in the tongue coating in patients with PMO of Gan-shen deficiency syndrome type. Therefore, it is rational that the tongue microbiome may reflect the health status of the human body.

In conclusion, we revealed the possible mechanisms underlying traditional tongue diagnosis and highlighted the potential for using tongue microbiome features to reflect the holistic status of the human body by studying the tongue microbiomes of patients with PMO and healthy women using bacterial analysis. Our current sample size was small however, and further studies with a greater sample size are required in order to gain a comprehensive understanding of this system. The observations described in this study may provide new insight into the biological basis of TCM tongue diagnosis.

\section{Acknowledgements}

This study was supported by the National Natural Science Foundation of China (grant nos. $81202645 \& 81173203$ ) and the Natural Science Foundation of Fujian Province (grant no. 2011J05076).

\section{References}

1. Ma T, Tan C, Zhang H, Wang M, Ding W and Li S: Bridging the gap between traditional Chinese medicine and systems biology: the connection of Cold Syndrome and NEI network. Mol Biosyst 6: 613-619, 2010.

2. Van der Sleen MI, Slot DE, Van Trijffel E, Winkel EG and Van der Weijden GA: Effectiveness of mechanical tongue cleaning on breath odour and tongue coating: a systematic review. Int J Dent Hyg 8: 258-268, 2010.

3. Van Tornout M, Dadamio J, Coucke W and Quirynen M: Tongue coating: related factors. J Clin Periodontol 40: 180-185, 2013.

4. Kim J, Han GJ, Choi BH, et al: Development of differential criteria on tongue coating thickness in tongue diagnosis. Complement Ther Med 20: 316-322, 2012.

5. Li S, Zhang ZQ, Wu LJ, Zhang XG, Li YD and Wang YY: Understanding ZHENG in traditional Chinese medicine in the context of neuro-endocrine-immune network. IET Syst Biol 1: 51-60, 2007.

6. Jiang M, Zha Q, Lu C, He Y and Lu A: Association between tongue appearance in Traditional Chinese Medicine and effective response in treatment of rheumatoid arthritis. Complement Ther Med 19: 115-121, 2011.

7. Feng Y, Xu H, Qu D, Zheng F, Shi DZ and Chen KJ: Study on the tongue manifestations for the blood-stasis and toxin syndrome in the stable patients of coronary heart disease. Chin J Integr Med 17: 333-338, 2011.

8. Yamada Y, Ando F and Shimokata H: Association of candidate gene polymorphisms with bone mineral density in communitydwelling Japanese women and men. Int J Mol Med 19: 791-801, 2007.

9. Liang W, Lin M, Li X, et al: Icariin promotes bone formation via the BMP-2/Smad4 signal transduction pathway in the hFOB 1.19 human osteoblastic cell line. Int J Mol Med 30: 889-895, 2012.
10. Shan PF, Wu XP, Zhang H, et al: Bone mineral density and its relationship with body mass index in postmenopausal women with type 2 diabetes mellitus in mainland China. J Bone Miner Metab 27: 190-197, 2009.

11. Kim J, Jung Y, Park K and Park JW: A digital tongue imaging system for tongue coating evaluation in patients with oral malodour. Oral Dis 15: 565-569, 2009.

12. Khandelwal S, Chandra M and Lo JC: Clinical characteristics, bone mineral density and non-vertebral osteoporotic fracture outcomes among post-menopausal U.S. South Asian Women. Bone 51: 1025-1028, 2012.

13. Mills JN, Valli VE and Lumsden JH: Cyclical changes of vaginal cytology in the cat. Can Vet J 20: 95-101, 1979.

14. Kazor CE, Mitchell PM, Lee AM, et al: Diversity of bacterial populations on the tongue dorsa of patients with halitosis and healthy patients. J Clin Microbiol 41: 558-563, 2003.

15. McGarr SE, Ridlon JM and Hylemon PB: Diet, anaerobic bacterial metabolism, and colon cancer: a review of the literature. J Clin Gastroenterol 39: 98-109, 2005.

16. Qi WJ, Zhang MM, Wang H, Wen Y, Wang BE and Zhang SW: Research on the relationship between thick greasy tongue fur formation and vascular endothelial cell permeability with the protein expression of zonula occludens-1. Chin J Integr Med 17: 510-516, 2011.

17. Kikutani T, Tamura F, Nishiwaki K, et al: The degree of tongue-coating reflects lingual motor function in the elderly. Gerodontology 26: 291-296, 2009.

18. O'Brien KA and Birch S: A review of the reliability of traditional East Asian medicine diagnoses. J Altern Complement Med 15: 353-366, 2009.

19. Tolentino Ede S, Chinellato LE and Tarzia O: Saliva and tongue coating $\mathrm{pH}$ before and after use of mouthwashes and relationship with parameters of halitosis. J Appl Oral Sci 19: 90-94, 2011.

20. Kato Y, Nagashima Y, Baba Y, et al: Expression of SPARC in tongue carcinoma of stage II is associated with poor prognosis: An immunohistochemical study of 86 cases. Int J Mol Med 16: 263-268, 2005.

21. Anastasi JK, Currie LM and Kim GH: Understanding diagnostic reasoning in TCM practice: tongue diagnosis. Altern Ther Health Med 15: 18-28, 2009.

22. Calil C, Liberato FL, Pereira AC, de Castro Meneghim M, Goodson JM and Groppo FC: The relationship between volatile sulphur compounds, tongue coating and periodontal disease. Int J Dent Hyg 7: 251-255, 2009.

23. Kislig K, Wilder-Smith CH, Bornstein MM, Lussi A and Seemann R: Halitosis and tongue coating in patients with erosive gastroesophageal reflux disease versus nonerosive gastroesophageal reflux disease. Clin Oral Investig 17: 159-165, 2013.

24. Zadik Y, Drucker S and Pallmon S: Migratory stomatitis (ectopic geographic tongue) on the floor of the mouth. J Am Acad Dermatol 65: 459-460, 2011.

25. Abe S, Ishihara K, Adachi M and Okuda K: Tongue-coating as risk indicator for aspiration pneumonia in edentate elderly. Arch Gerontol Geriatr 47: 267-275, 2008.

26. Takeshita T, Tomioka M, Shimazaki Y, et al: Microfloral characterization of the tongue coating and associated risk for pneumonia-related health problems in institutionalized older adults. J Am Geriatr Soc 58: 1050-1057, 2010.

27. Takeshita T, Suzuki N, Nakano Y, et al: Relationship between oral malodor and the global composition of indigenous bacterial populations in saliva. Appl Environ Microbiol 76: 2806-2814, 2010.

28. Kamaraj DR, Bhushan KS, Laxman VK and Mathew J: Detection of odoriferous subgingival and tongue microbiota in diabetic and nondiabetic patients with oral malodor using polymerase chain reaction. Indian J Dent Res 22: 260-265, 2011.

29. Dewhirst FE, Chen T, Izard J, et al: The human oral microbiome. J Bacteriol 192: 5002-5017, 2010.

30. Kishi M, Ohara-Nemoto Y, Takahashi M, Kishi K, Kimura S and Yonemitsu M: Relationship between oral status and prevalence of periodontopathic bacteria on the tongues of elderly individuals. J Med Microbiol 59: 1354-1359, 2010. 